\title{
Atuação da equipe multidisciplinar na terapia nutricional de pacientes sob cuidados intensivos
}

\author{
The action of a multidisciplinary team in the \\ nutritional care of critically ill patients
}

Heitor Pons LEITE 1,2

Werther Brunow de CARVALHO ${ }^{1,2}$

Juliana Fernandez SANTANA e MENESES ${ }^{3,4}$

\section{R E S U M O}

Pacientes hospitalizados podem ter necessidades nutricionais especiais em função da desnutrição e dos desequilíbrios metabólicos impostos pelas doenças. A terapia nutricional, principalmente nos estágios críticos das enfermidades, deve ser administrada de modo seguro e eficaz. Vários estudos têm avaliado o papel da equipe multidisciplinar na administração da terapia nutricional. Com o objetivo de identificar os problemas inerentes à administração da terapia nutricional em pacientes hospitalizados e verificar o impacto da atuação de uma equipe multidisciplinar na qualidade dos procedimentos, foi realizada uma revisão que analisou artigos publicados entre 1980 e 2004 sobre o papel da atuação de equipes multidisciplinares no cuidado e na evolução nutricional de pacientes hospitalizados, principalmente os que se encontravam sob cuidados intensivos. Os termos utilizados na pesquisa foram: "multidisciplinary team", "nutritional support", "parenteral nutrition", "enteral feeding", "critically ill", "intensive care unit", "critically ill child". Dos 130 estudos inicialmente identificados, foram selecionados 24 , dos quais 14 compararam o padrão de terapia nutricional com e sem a presença da equipe multidisciplinar. Os principais problemas detectados na administração de terapia nutricional em pacientes hospitalizados foram a oferta inadequada de nutrientes, as complicações infecciosas e metabólicas e o uso excessivo de nutrição parenteral. Nos estudos comparativos, a presença da equipe multidisciplinar melhorou o padrão de oferta nutricional, reduziu a incidência de complicações e os custos.

Termos de indexação: apoio nutricional, desnutrição, nutrição enteral, nutrição parenteral, unidade de cuidados intensivos.

\footnotetext{
1 Departamento de Pediatria, Disciplina de Nutrologia, Universidade Federal de São Paulo. Rua Loefgreen, 1647, 04040-032, São Paulo, SP, Brasil. Correspondência para/Correspondence to: H.P. LEITE. E-mail: <bri1259@terra.com.br>.

${ }^{2}$ Unidade de Cuidados Intensivos Pediátricos, Hospital São Paulo. São Paulo, SP, Brasil.

3 Equipe Multidisciplinar de Terapia Nutricional, Hospital São Paulo. São Paulo, SP, Brasil.

${ }^{4}$ Mestranda, Disciplina de Nutrologia, Departamento de Pediatria, Universidade Federal de São Paulo. São Paulo, SP, Brasil.
} 


\section{A B S T R A C T}

Hospitalized patients may have special nutrient requirements imposed by a combination of malnutrition and enhanced utilization of nutrients resulting from the disease process. Nutritional support, mainly during critical stages of disease, should be provided safely and effectively. Several studies have evaluated the paper of a multidisciplinary team in the administration of a nutritional therapy. Individually, the majority of these studies are underpowered to evaluate an effect on the quality of nutritional care. With the objective to identify problems inherent to the supply of nutritional support to hospitalized patients and verify the impact of the actions of a multidisciplinary team on the quality of these procedures, we analysed articles that have been published between 1980 and 2004 about the role of the action of multidisciplinary teams in the care and nutritional outcome of hospitalized patients, especially those undergoing intensive care. The terms used for the search were: "multidisciplinary team", "nutritional support", "parenteral nutrition", "enteral feeding", "critically ill", intensive care unit, "critically ill child". Of 130 studies, intially identified, just 24 were selected, of which 14 compared the standard of nutritional therapy with and without the presence of a multidisciplinary team. The inadequate supply of nutrients, infection and metabolic complications and the excessive use of parenteral nutrition were the main problems detected in the supply of nutritional support to hospitalized patients. In the comparative studies, the presence of the multidisciplinary team improved the pattern of nutritional support, and reduced the incidence of complications and the costs.

Indexing terms: nutritional support, malnutrition, enteral nutrition, parenteral nutrition, intensive care unit.

\section{N T R O D U Ç Ã O}

Mesmo com os avanços da terapia nutricional e metabólica nas últimas décadas, a desnutrição continua sendo comum em pacientes hospitalizados, com prevalência variando entre $30 \%$ e $65 \%$ nos diferentes estudos ${ }^{1-3}$, e podendo estar presente no momento da admissão hospitalar ou desenvolver-se no decorrer da internação.

Em estudo realizado pela Sociedade Brasileira de Nutrição Parenteral e Enteral (SBNPE), observou-se que aproximadamente $30 \%$ dos pacientes hospitalizados tornavam-se desnutridos nas primeiras 48 horas de internação. Em três a sete dias esse porcentual aumentava em 15\%, chegando a $60 \%$ depois de quinze dias de internação².

Pacientes com infecções graves, traumatismos ou em pós-operatório de grandes cirurgias são particularmente vulneráveis a desenvolver desnutrição. Ingestão diminuída, restrição de oferta hídrica, instabilidade hemodinâmica, diminuição da absorção e interação droga-nutriente ${ }^{3}$ podem ser situações de risco nutricional ${ }^{1}$. Além desses fatores, a pouca atenção dos profissionais de saúde ao cuidado nutricional - levando à indicação inadequada, à falta de avaliação nutricional e à monitoração pouco freqüente - é comumente observada e pode contribuir para a desnutrição ${ }^{1-3}$.

Dada a complexidade dos fatores envolvidos na monitoração do paciente hospitalizado e no tratamento da desnutrição hospitalar, a formação de uma equipe multidisciplinar pode ser fundamental para assegurar atenção adequada aos pacientes hospitalizados ${ }^{4-6}$. O trabalho conjunto de especialistas com formações distintas permite integrar, harmonizar e complementar os conhecimentos e habilidades dos integrantes da equipe para cumprir o objetivo proposto, que é o de identificar, intervir e acompanhar o tratamento dos distúrbios nutricionais ${ }^{4}$.

Atualmente, a Agência Nacional de Vigilância Sanitária (ANVISA) regulamenta a formação de Equipe Multidisciplinar de Terapia Nutricional (EMTN), obrigatória nos hospitais brasileiros. Essa regulamentação é regida pelas portarias 272 (Regulamento Técnico de Terapia de Nutrição Parenteral) e 337 (Regulamento Técnico de Terapia de Nutrição Enteral). Fazem parte das atribuições da EMTN: definir metas técnico-administrativas, realizar triagem e vigilância nutricional, avaliar o estado nutricional, indicar terapia nutricional e metabólica, assegurar 
condições ótimas de indicação, prescrição, preparação, armazenamento, transporte, administração e controle dessa terapia; educar e capacitar a equipe; criar protocolos, analisar o custo e o benefício e traçar metas operacionais da EMTN ${ }^{7,8}$.

Na maioria dos hospitais brasileiros, a EMTN funciona como uma equipe de apoio, ou seja, a equipe assistencial conduz o doente, e a EMTN, por sua vez, estabelece diretrizes gerais e protocolos de conduta nutricional. Em outros hospitais, a EMTN tem atuação clínica avaliando diretamente os doentes mediante solicitação da equipe assistencial.

Em 1991, a ASPEN (Sociedade Americana de Nutrição Enteral e Parenteral) pesquisou 1680 hospitais nos Estados Unidos, encontrando a presença de EMTN em apenas $29,0 \%$ deles. Um estudo realizado em 833 hospitais na Alemanha verificou que 5,6\% contavam com a atuação da EMTN de forma satisfatória. No Reino Unido, a EMTN estava presente em 37,3\% dos hospitais. No Brasil, a SBNPE, em 1997, pesquisando 232 hospitais dos estados de São Paulo e Rio de Janeiro, observou que apenas 20,0\% tinham EMTN formada 5 .

Ao observar a importância da terapia nutricional em crianças internadas em Unidade de Cuidados Intensivos Pediátricos (UCIP), em 1992, um médico especialista na área iniciou o acompanhamento nutricional dos pacientes gravemente doentes no Hospital São Paulo. A partir de 1993, instituiu-se um programa de educação continuada para residentes e em 2001 foi implantada a equipe multidisciplinar com médicos e nutricionistas para o acompanhamento diário e sistemático da evolução nutricional dos pacientes internados na unidade.

Por meio de revisão criteriosa da literatura, procurou-se identificar os problemas inerentes à administração da terapia nutricional aos pacientes hospitalizados e verificar o impacto da atuação da equipe multidisciplinar na qualidade desse procedimento.

Foram utilizadas as bases de dados Pubmed e Lilacs, para revisão bibliográfica, tendo sido pesquisados estudos publicados de 1980 a 2004 referentes à atuação da equipe multidisciplinar em hospitais e principalmente em pacientes sob cuidados intensivos. Procurou-se identificar estudos que comparassem grupos de pacientes com e sem monitoração de equipe multidisciplinar e os que relatavam a atuação dessa equipe na área clínica. Os termos utilizados para a busca foram: "multidisciplinary team", "nutritional support", "parenteral nutrition", "enteral feeding", "critically ill", "intensive care unit", "critically ill child".

\section{RESULTADOSE DISCUSSÃ O}

Foram identificados inicialmente 130 estudos, dos quais foram selecionados 24 por atenderem aos critérios desta pesquisa. Desses, 14 eram estudos comparativos e somente um foi feito com pacientes pediátricos.

Os estudos comparativos relatando o impacto da EMTN na qualidade da terapia nutricional e metabólica estão nos Anexos 1 e 2 .

O suporte nutricional e metabólico adequado, principalmente em pacientes sob cuidados intensivos exige um planejamento que deve incluir avaliação e monitoramento sistemáticos do estado metabólico e nutricional. Contudo, freqüentemente são observadas falhas nesse aspecto. Estudos revelam que, quando não há monitoramento por EMTN a avaliação nutricional é feita em 3\% a 7\% dos pacientes hospitalizados. Já na presença dessa equipe, a avaliação ocorre em $37 \%$ a $68 \%$ dos doentes ${ }^{9-11}$.

Tais pacientes apresentam limitações de ingestão e absorção dos nutrientes, o que os coloca em risco nutricional. Existem métodos para repor ou suplementar as necessidades individuais, porém, quando usados de maneira imprópria, podem levar a complicações metabólicas, infecciosas e mecânicas. Os trabalhos que compararam a administração da terapia nutricional e metabólica, com ou sem a presença de uma equipe multidisciplinar, mostraram diminuição significante de complicações metabólicas ${ }^{9,12-15}$, 
redução da incidência de infecção ${ }^{10,12,14-16}$ e das complicações mecânicas ${ }^{12,14-16}$.

Além de redução das complicações, alguns estudos mostraram que a presença de equipe multidisciplinar aumentou a freqüência de avaliação nutricional $\left.\right|^{9,10,17,18}$, proporcionou oferta mais adequada de nutrientes ${ }^{18}$, indicação mais apropriada de NP ${ }^{19,20}$ e diminuição de custos ${ }^{9,19-21 .}$.

Os estudos que relataram a diminuição do uso de NP e de custos não discriminaram se os pacientes estavam sob cuidados intensivos ou não9,19,20, e não informaram sobre as características clínicas dos pacientes estudados ${ }^{21}$, impedindo uma melhor conclusão sobre os resultados obtidos.

Para avaliar a eficácia do trabalho multidisciplinar em um hospital britânico, Newton acompanhou durante um ano a qualidade no cuidado nutricional e a presença de complicações, a partir da formação de uma equipe multidisciplinar. Nesse período foi observada redução significativa dos custos da NP, assim como de perdas por bolsas desprezadas e diminuição de prescrição inapropriada da TNP. Outro dado relevante deste estudo foi a redução de complicações infecciosas. Provavelmente isso ocorreu devido ao fato da NP ter sido administrada em via exclusiva e também ter sido monitorada por um membro treinado da equipe. Programas de educação continuada foram ministrados, podendo ser esse outro fator que contribuiu para os resultados favoráveis ${ }^{22}$.

Em estudo retrospectivo e multicêntrico em hospitais alemães, avaliou-se a prevalência e a estrutura da EMTN e verificou-se se havia diferença em relação à terapia nutricional entre os que contavam e os que não contavam com a equipe multidisciplinar. Concluiu-se que há baixa prevalência de EMTN na Alemanha e que nos hospitais que contavam com essas equipes houve redução do tempo de internação, da incidência de complicações, redução de custos, maior uso de nutrição enteral em relação à parenteral e redução da morbidade ${ }^{8}$.

Embora tenha havido progresso na qualidade da terapia nutricional ao longo do tempo, estudos revelam que a abordagem multidisciplinar tem se restringido principalmente à população adulta. Em crianças, especialmente no período neonatal, há maior necessidade de $\mathrm{NP}$, atraso de crescimento secundário à prematuridade e intolerância à NE, fatores que tornam preocupante a ausência do cuidado multidisciplinar ${ }^{11}$.

No único estudo pediátrico comparativo levantado, a avaliação do padrão da terapia nutricional e metabólica em pacientes internados na unidade de cuidados intensivos de um hospital-escola detectou deficiências como falta de avaliação nutricional e metabólica, oferta inadequada de macro e micronutrientes e uso preferencial da via parenteral em relação à enteral, sendo na ocasião sugerida a criação de uma equipe multidisciplinar ${ }^{1}$. Após um programa de educação continuada para os médicos residentes, essas deficiências foram parcialmente solucionadas ${ }^{17}$, mas ainda assim foi reforçada a necessidade do acompanhamento multiprofissional.

Em que pesem os efeitos benéficos da equipe multidisciplinar na qualidade da terapia nutricional, nem todos os hospitais contam com uma equipe atuante. Estudos em países europeus têm revelado pouca aceitação da EMTN nos hospitais, fato atribuído ao possível aumento dos custos hospitalares ${ }^{2,8}$. Por outro lado, há evidências recentes que sugerem que a atuação de uma equipe multidisciplinar na nutrição parenteral é um fator de redução de custos hospitalares ${ }^{23}$.

Outro estudo retrospectivo em adultos que receberam NP durante sete meses comparou a qualidade da NP com e sem a presença de uma EMTN. Os resultados desse estudo revelaram redução significante de complicações por cateter venoso, maior freqüência de avaliação nutricional, uso do balanço nitrogenado e maior número de pacientes com necessidades nutricionais alcançadas $^{10}$. Não houve diferença para complicações metabólicas e em relação à mortalidade. Alguns desses resultados podem não ser fidedignos, pois os grupos comparados eram heterogêneos do ponto de vista nutricional e, além 
disso, dizem respeito a pacientes gravemente doentes.

É oportuno ressaltar que a atuação dessa equipe nos hospitais em que a EMTN tem atuação direta no acompanhamento dos doentes, principalmente na área específica de cuidados intensivos, é um fato inovador e requer tempo para adaptação do corpo clínico envolvido no cuidado dos pacientes. Inicialmente podem ocorrer problemas de aceitação da EMTN por parte da própria equipe assistencial, o que pode prejudicar o trabalho e a qualidade da atenção ao doente. Para a atuação ser efetiva, é necessária a convivência harmoniosa entre essa equipe e o corpo clínico e assistencial. Por vezes, a equipe assistencial, responsabilizada pelo acompanhamento diário dos pacientes, pode se sentir insatisfeita frente às mudanças nas rotinas. Esse seria um dos motivos para a não aceitação da EMTN.

A pouca informação sobre nutrição durante a graduação profissional pode acarretar desinteresse por essa área e provavelmente aumentar a chance de indicação inadequada da terapia nutricional. Essa observação pode ser estendida ao nutricionista, que habitualmente não tem experiência com o uso de NP. Há também deficiência quantitativa no quadro de nutricionistas atuando na área clínica, com conseqüente prejuízo na assistência nutricional.

Pesquisa feita pela British Association of Parenteral Enteral Nutrition (BAPEN) em 1996 revelou que apenas $29 \%$ dos farmacêuticos têm atuação na área de nutrição clínica. Essa deficiência pode, por sua vez, favorecer o aumento da ocorrência de incompatibilidades droga-nutriente, evento que exige atenção especial, principalmente em pacientes que estão em uso concomitante de diversos medicamentos. A incompatibilidade droga-nutriente pode diminuir a absorção ou até inativar medicamentos ${ }^{24}$.

Há que se considerar também a pouca ênfase ao trabalho em equipe durante a graduação e pós-graduação; o trabalho em conjunto deveria ser mais explorado ao formar os profissionais que irão para o ensino e para a assistência ${ }^{24,25}$. Ao perceber a necessidade de profissionais aptos a exercerem a prática clínica, relacionando-a aos avanços da pesquisa, uma universidade americana criou o doutorado em nutrição clínica. O programa de doutorado habilita o pós-graduando a trabalhar na prática clínica juntamente à pesquisa ${ }^{25}$. Essa estratégia poderia melhorar os modelos assistenciais que priorizam o trabalho em equipe, refletindo no ensino, pesquisa e na assistência em geral.

Embora alguns estudos tenham confirmado a necessidade e os benefícios da presença da EMTN na terapia nutricional de pacientes hospitalizados ${ }^{11,26}$, as dificuldades na implantação dessa equipe têm sido a falta de informação e de recursos disponíveis, as questões de política hospitalar e a pouca aceitação por parte da equipe assistencial, além das próprias dificuldades relacionadas à gravidade dos pacientes em estado crítico.

Esta revisão da literatura permitiu concluir que há falhas na terapia nutricional de pacientes hospitalizados e que a presença de uma equipe multidisciplinar pode reduzir as deficiências nutricionais, as complicações e os custos.

O processo de implantação dessa equipe deve ser gradual e as dificuldades deverão ser resolvidas pela informação e pela educação continuada em nutrição. As informações devem ser transmitidas e trabalhadas em conjunto com os profissionais das diversas áreas, de modo a favorecer a integração de todos os envolvidos no cuidado do paciente. A aplicação de protocolos, a integração e a colaboração entre os membros da equipe multidisciplinar e a equipe assistencial já existente no serviço na solução dos problemas devem se incorporar à rotina de trabalho, contribuindo assim para a melhoria na qualidade da assistência prestada aos doentes.

\section{REFERÊ NCIAS}

1. Leite HP, Iglesias SBO, Faria CMS, Carvalho WB. Nutritional and metabolic assessment of critically ill children. Rev Ass Med Brasil. 1996; 42(4): 215-21. 
2. Waitzberg DL, Caiaffa WT, Correia MI. Hospital malnutrition: the Brazilian national survey (IBRANUTRI): a study of 4000 patients. Nutrition. 2001; 17(7-8):573-80.

3. Han-Markey TL, Wesley JR. Pediatric Critical Care. American Society for Parenteral and Enteral Nutrition. 1999. Chap. 34:34.1-34.10

4. Penié JB, Porbén SS, Gonzaléz CM, Ibarra AMS. Grupo de apoyo nutricional hospitalario: diseño, composición y programa de actividades. Rev Cubana Aliment Nutr. 2000; 14(1):55-64.

5. Silva MLT. A Importância da equipe multiprofissional em terapia nutriticional. In: Watzberg DL. Nutricão oral, enteral e parenteral na prática clínica. São Paulo: Atheneu; 2000. p.1627-34.

6. Nehme AE. Nutritional support of the hospitalized patient. JAMA. 1980; 43(19):1906-8.

7. Brasil. Ministério da Saúde. Agência Nacional de Vigilância Sanitária, RDC n63, de 6 de julho de 2000. Aprova o regulamento técnico para fixar requisitos mínimos exigidos para a terapia de nutrição enteral. Diário Oficial da União; Poder Executivo, de 7 de julho de 2000. Revoga a portaria n 337 de 14 de abril de 1999.

8. Senkal M, Dormann A, Stehle P, Shang E, Suchner $U$. Survey on structure and performance of nutrition-support teams in germany. Clin Nutr. 2002; 21(4):329-35.

9. ChrisAnderson D, Heimburger DC, Morgan SL, Geels WJ, Henry KL, Conner W, et al. Metabolic complications of total parenteral nutrition: effects of a nutrition support service. JPEN. 1996; 20(3):206-10.

10. PNG DJC, Ong C, Chan S. Surgical nutritional team and its impact on total parenteral nutrition in the National University Hospital, Singapore. Int J Clin Pract. 1997; 51(6):350-2.

11. Mayfield SR, Albrecht J, Roberts L, Lair C. The role of the nutritional support team in neonatal intensive care. Semin Perinatol. 1989; 13(2): 88-96.

12. Fettes SB, Lough M. An audit of the provision of parenteral nutrition in two acute hospitals: team versus non-team. Scot Med J. 2000; 45(4):121-5.

13. Newton R, Timmis L, Bowling TE. Changes in parenteral nutrition supply when the nutrition support team controls prescribing. Nutrition. 2001; 17(4):347-50.

14. Brown RO, Carlson SD, Cowan GSM, Powers DA, Luther RW. Enteral nutritional support management in a University Teaching Hospital: team vs nonteam. JPEN. 1987; 11(1):52-6.
15. O'Brien DD, Hodges RE, Day AT, Waxman KS, Rebello T. Recommendations of nutriton support team promote cost containment. JPEN. 1986; 10(3):300-2.

16. Dalton MJ, Schepers G, Gee JP, Alberts CC, Eckhauser FE, Kirking DM. Consultative total parenteral nutrition teams: the effect on the incidence of total parenteral nutrition-related complications. JPEN. 1984; 8(2):146-52.

17. Leite HP, Iglesias SBO, Faria CMS, Ikeda AM, Albuquerque MP, Carvalho WB. Evolution of the nutritional support pattern in pediatric intensive care. São Paulo Med J. 1998; 116(1):1606-12.

18. Goldstein M, Braitman LE, Levine GM. The medical and financial costs associated with termination of a nutrition support nurse. JPEN. 2000; 24(6): 323-7.

19. Schwartz DB. Enhanced enteral and parenteral nutrition practice and outcomes in a $\mathrm{n}$ intensive care unit with a hospital-wide performance improvement process. J Am Diet Assoc. 1996; 96(5):484-9.

20. Traeger SM, Williams GB, Milliren G, Young DS, Fisher $M$, Haug T. Total parenteral nutrition by a nutrition support team: improved quality of care. JPEN. 1986; 10(4):408-12.

21. Gales BJ, Riley DG. Improved total parenteral nutrition therapy management by a nutritional support team. Hosp Pharm. 1994; 29(5):469-70, 473-5.

22. Powers DA, Brown RO, Cowan GSM, Luther RW, Sutherland DA, Drexler PG. Nutritional support team vs nonteam management of enteral nutritional support in a veterans administration medical center teaching hospital. JPEN. 1986; 10(6):635-8.

23. Naylor CJ, Griffiths RD, Fernandez RS. Does a multidisciplinary total parenteral nutrition team improve patient outcomes? A systematic review. JPEN. 2004; 28(4):251-8.

24. Pennington CR. Current perspectives on parenteral nutrition in adults. Maidenhead: BAPEN; 1996.

25. Touger-Decker R. The new advanced clinical nutrition degree: the practice doctorate in clinical nutrition. Nutrition. 2004; 20(5):494-5.

26. Gales BJ, Gales MJ. Nutritional support seams: a review of comparative trials. Ann Pharmacother. 1994; 28(2):227-35.

27. Maurer J, Weinbaum F, Turner J, Brady T, Pistone B, D'Addario $V$, et al. Reducing the Inappropriate Use of The Parenteral Nutrition in na Acute Care Teaching Hospital. JPEN. 1996; 20(4):272-4.

Recebido para publicação em 25 de setembro de 2003 e aceito em 23 de agosto de 2004. 


\section{ANEXO 1}

ESTUDOS COMPARATIVOS SOBRE A ATUAÇÃO DA EQUIPE MULTIDISCIPLINAR NA TERAPIA NUTRICIONAL E METABÓLICA

\begin{tabular}{|c|c|c|c|}
\hline Estudos & Participantes & Métodos & Resultados \\
\hline $\begin{array}{l}\text { Nehme }^{6} \\
(1980)\end{array}$ & $\begin{array}{l}375 \text { pacientes adultos recebendo } \\
\text { NP. }\end{array}$ & $\begin{array}{l}\text { Estudo prospectivo comparando } 2 \\
\text { grupos: } \\
\text { A: monitorado por EMTN. } \\
\text { B: não monitorado por EMTN. }\end{array}$ & $\begin{array}{l}\text { O grupo B apresentou: } \\
\text { - maior incidência de compli- } \\
\text { cações metabólicas, infeccio- } \\
\text { sas e mecânicas, } \\
\text { - menor freqüência de avalia- } \\
\text { ção nutricional. }\end{array}$ \\
\hline $\begin{array}{l}\text { Powers et al. }{ }^{22} \\
(1986)\end{array}$ & $\begin{array}{l}101 \text { pacientes adultos recebendo } \\
\text { NE durante } 5 \text { meses. }\end{array}$ & $\begin{array}{l}\text { Estudo prospectivo comparando } \\
\text { pacientes com monitoração de } \\
\text { EMTN }(n=50) \text { e em monitoração de } \\
\text { EMTN }(n=51) \text {. }\end{array}$ & $\begin{array}{l}\text { Monitoração da EMTN: } \\
\text { - reduziu complicações mecâ- } \\
\text { nicas, metabólicas, infeccio- } \\
\text { sas, } \\
\text { - aumentou número de pacien- } \\
\text { tes com necessidades nutri- } \\
\text { cionais contempladas, } \\
\text { - houve maior uso de balanço } \\
\text { nitrogenado. }\end{array}$ \\
\hline $\begin{array}{l}\text { ChrisAnderson et al. }{ }^{9} \\
\text { (1995) }\end{array}$ & $\begin{array}{l}363 \text { pacientes adultos clínicos e } \\
\text { cirúrgicos recebendo NP. }\end{array}$ & $\begin{array}{l}\text { Estudo prospectivo comparando o } \\
\text { padrão da TN sem equipe multidis- } \\
\text { ciplinar (1979) e posteriormente com } \\
\text { equipe multidisciplinar (1992). }\end{array}$ & $\begin{array}{l}\text { No grupo de pacientes moni- } \\
\text { torados por EMTN houve re- } \\
\text { dução de complicações meta- } \\
\text { bólicas (hiperglicemia e hipofos- } \\
\text { fatemia). }\end{array}$ \\
\hline $\begin{array}{l}\text { Schwartz }{ }^{19} \\
(1996)\end{array}$ & $\begin{array}{l}\text { Pacientes adultos críticos e cirúrgi- } \\
\text { cos que utilizaram NE ou NP por } \\
2 \text { meses. }\end{array}$ & $\begin{array}{l}\text { Estudo prospectivo que avaliou a TN } \\
\text { antes e após } 2 \text { anos de programas } \\
\text { educacionais e criação de equipe } \\
\text { multidisciplinar. }\end{array}$ & $\begin{array}{l}\text { Necessidades energético-pro- } \\
\text { téicas atingidas em maior nú- } \\
\text { mero de pacientes e aumento } \\
\text { do uso da NE. }\end{array}$ \\
\hline $\begin{array}{l}\text { Maurer et al. }{ }^{27} \\
(1996)\end{array}$ & $\begin{array}{l}50 \text { pacientes adultos clínicos e ci- } \\
\text { rúrgicos que receberam NP. }\end{array}$ & $\begin{array}{l}\text { Estudo prospectivo com formação } \\
\text { de EMTN para avaliar o uso da NP. }\end{array}$ & $\begin{array}{l}\text { Após criação da equipe houve } \\
\text { diminuição de custos e de uso } \\
\text { inapropriado da NP. }\end{array}$ \\
\hline $\begin{array}{l}\text { Fettes et al. }{ }^{12} \\
(2000)\end{array}$ & $\begin{array}{l}47 \text { pacientes adultos recebendo } \\
\text { NP. }\end{array}$ & $\begin{array}{l}\text { Estudo prospectivo que comparou } \\
\text { a TN em } 2 \text { hospitais. Um com moni- } \\
\text { toração da EM e outro sem a pre- } \\
\text { sença da EM. }\end{array}$ & $\begin{array}{l}\text { No hospital com EMTN, a } \\
\text { maioria dos pacientes tiveram } \\
\text { as necessidades nutricionais } \\
\text { estimadas, redução de custos, } \\
\text { e de complicações metabólicas. }\end{array}$ \\
\hline $\begin{array}{l}\text { Newton }^{13} \\
(2001)\end{array}$ & $\begin{array}{l}\text { Pacientes adultos, hospitalizados, } \\
\text { recebendo NP. }\end{array}$ & $\begin{array}{l}\text { Avaliou-se a NP durante um ano } \\
\text { após a formação da EMTN. }\end{array}$ & $\begin{array}{l}\text { - redução de custos } \\
\text { - redução de prescrição inade- } \\
\text { quada. } \\
\text { - redução de complicações in- } \\
\text { fecciosas. }\end{array}$ \\
\hline
\end{tabular}

$\mathrm{TN}=$ terapia nutricional; $\mathrm{NE}=$ nutrição enteral; $\mathrm{NP}=$ nutrição parenteral. 
ANEXO 2

ESTUDOS COMPARATIVOS SOBRE A ATUAÇÃO DA EQUIPE MULTIDISCIPLINAR NA TERAPIA NUTRICIONAL E METABÓLICA

\begin{tabular}{|c|c|c|c|}
\hline Estudos & Participantes & Métodos & Resultados \\
\hline $\begin{array}{l}\text { Dalton et al. }{ }^{16} \\
(1984)\end{array}$ & $\begin{array}{l}\text { Adultos recebendo NP: } \\
\text { Grupo A: } 28 \text { pacientes controle. } \\
\text { Grupo B: } 29 \text { pacientes monitorados } \\
\text { pela EMTN. }\end{array}$ & $\begin{array}{l}\text { Estudo prospectivo avaliando o pa- } \\
\text { drão de TN com e sem EMTN. }\end{array}$ & $\begin{array}{l}\text { No grupo que recebeu mo- } \\
\text { nitoração houve redução de } \\
\text { complicações metabólicas e } \\
\text { infecciosas. }\end{array}$ \\
\hline $\begin{array}{l}\text { O'Brien et al. }{ }^{15} \\
(1986)\end{array}$ & $\begin{array}{l}\text { Pacientes que receberam NP ou NE } \\
(n=31) \text { durante o período de } \\
\text { dezesseis meses. }\end{array}$ & $\begin{array}{l}\text { Estudo retrospectivo comparando a } \\
\text { diferença de custo e a adequação } \\
\text { da TN quando iniciada por EMTN e } \\
\text { quando pela própria equipe } \\
\text { assistencial. }\end{array}$ & $\begin{array}{l}\text { A presença da EMTN aumen- } \\
\text { tou o uso da NE e diminuiu } \\
\text { os custos da TN. }\end{array}$ \\
\hline $\begin{array}{l}\text { Traeger et al. }{ }^{20} \\
(1986)\end{array}$ & $\begin{array}{l}\text { Todos os pacientes adultos (69) que } \\
\text { receberam NP durante sete meses. }\end{array}$ & $\begin{array}{l}\text { Estudo retrospectivo que comparou } \\
\text { o padrão da NP com e sem acompa- } \\
\text { nhamento de EMTN. }\end{array}$ & $\begin{array}{l}\text { No grupo com EMTN hou- } \\
\text { ve: avaliação nutricional em } \\
\text { todos os pacientes, balanço } \\
\text { nitrogenado feito com } \\
\text { maior freqüência e houve } \\
\text { também redução das com- } \\
\text { plicações infecciosas. }\end{array}$ \\
\hline $\begin{array}{l}\text { Brown et al. }{ }^{14} \\
(1987)\end{array}$ & $\begin{array}{l}\text { Pacientes adultos }(n=102) \text { receben- } \\
\text { do NE durante três meses e meio. }\end{array}$ & $\begin{array}{l}\text { Estudo prospectivo comparando } \\
\text { pacientes com e sem monitoração } \\
\text { de EMTN. }\end{array}$ & $\begin{array}{l}\text { Os pacientes monitorados } \\
\text { por EM tiveram necessidades } \\
\text { nutricionais satisfeitas e re- } \\
\text { dução de complicações me- } \\
\text { cânicas, metabólicas e infec- } \\
\text { ciosas. }\end{array}$ \\
\hline $\begin{array}{l}\text { Gales et al. }{ }^{21} \\
(1994)\end{array}$ & $\begin{array}{l}\text { Pacientes adultos }(n=28) \text { que rece- } \\
\text { beram NP durante duas semanas: } \\
\text { Com EMTN: } 11 \text { pacientes. } \\
\text { Grupo Controle: } 17 \text { pacientes. }\end{array}$ & $\begin{array}{l}\text { Estudo prospectivo avaliando o pa- } \\
\text { drão de TN com e sem EMTN. }\end{array}$ & $\begin{array}{l}\text { No grupo monitorado por } \\
\text { EMTN houve melhor plane- } \\
\text { jamento nutricional e maior } \\
\text { oferta energético protéica. }\end{array}$ \\
\hline $\begin{array}{l}\text { PNG et al. }{ }^{10} \\
(1997)\end{array}$ & $\begin{array}{l}\text { Pacientes cirúrgicos (54) durante } 6 \\
\text { meses antes da formação da EMTN } \\
\text { e (43) após a formação da EMTN. }\end{array}$ & $\begin{array}{l}\text { Estudo de coorte histórico } \\
\text { prospectivo comparando o padrão } \\
\text { da TN antes e depois da formação } \\
\text { da EMTN. }\end{array}$ & $\begin{array}{l}\text { Após formação da EMTN } \\
\text { houve maior adequação da } \\
\text { TN e diminuição das compli- } \\
\text { cações mecânicas e infeccio- } \\
\text { sas. }\end{array}$ \\
\hline $\begin{array}{l}\text { Leite et al. }{ }^{17} \\
\text { (1998) }\end{array}$ & $\begin{array}{l}\text { Todas as crianças ( } 72) \text { que recebe- } \\
\text { ram TN durante no mínimo } 5 \text { dias: } \\
\text { F ase I- } 37 \text { crianças. } \\
\text { Fase II- } 35 \text { crianças após programa } \\
\text { de educação. }\end{array}$ & $\begin{array}{l}\text { Coorte histórico que avaliou a TN } \\
\text { em crianças internadas em UCI an- } \\
\text { tes e depois de um programa de } \\
\text { educação continuada. }\end{array}$ & $\begin{array}{l}\text { Após o programa houve } \\
\text { aumento do uso de NE, ade- } \\
\text { quação da oferta de energia } \\
\text { e micronutrientes e aumen- } \\
\text { to da freqüência de avalia- } \\
\text { ção nutricional. }\end{array}$ \\
\hline
\end{tabular}

$\mathrm{TN}=$ terapia nutricional; $\mathrm{NE}=$ nutrição enteral; $\mathrm{NP}=$ nutrição parenteral. 